\title{
Conserved developmental expression of Fezf in chordates and Drosophila and the origin of the Zona Limitans Intrathalamica (ZLI) brain organizer
}

Manuel Irimia ${ }^{1 *}$, Cristina Piñeiro², Ignacio Maeso ${ }^{1}$, José Luis Gómez-Skarmeta ${ }^{2 *}$, Fernando Casares ${ }^{2^{*}}$, Jordi Garcia-Fernàndez ${ }^{1 *}$

\begin{abstract}
Background: The zona limitans intrathalamica (ZLI) and the isthmus organizer (IsO) are two major secondary organizers of vertebrate brain development. These organizers are located at the interface of the expression domains of key patterning genes (Fezf-Irx and Otx-Gbx, respectively). To gain insights into the evolutionary origin of the ZLI, we studied Fezf in bilaterians.

Results: In this paper, we identified a conserved sequence motif (Fezf box) in all bilaterians. We report the expression pattern of Fezf in amphioxus and Drosophila and compare it with those of Gbx, Otx and Irx. We found that the relative expression patterns of these genes in vertebrates are fully conserved in amphioxus and flies, indicating that the genetic subdivisions defining the location of both secondary organizers in early vertebrate brain development were probably present in the last common ancestor of extant bilaterians. However, in contrast to vertebrates, we found that Irx-defective flies do not show an affected Fezf expression pattern.

Conclusions: The absence of expression of the corresponding morphogens from cells at these conserved genetic boundaries in invertebrates suggests that the organizing properties might have evolved specifically in the vertebrate lineage by the recruitment of key morphogens to these conserved genetic locations.
\end{abstract}

\section{Background}

Secondary morphogenetic organizers are located at the boundaries of major vertebrate brain compartments, and play essential roles in the development of the highly complex vertebrate brain. The two main brain internal organizers are the isthmus organizer ( $\mathrm{IsO}$ ) and the zona limitans intrathalamica (ZLI). The IsO is located in the midbrain-hindbrain boundary (MHB), at the abutting expression domains of Otx and Gbx, and the ZLI develops within the diencephalon, between the prethalamus and thalamus, at the boundary of Fezf and Irx gene expression domains (Figure 1A). As is typical for organizers, cells from these structures are the source of

\footnotetext{
* Correspondence: mirimia@gmail.com; jlgomska@upo.es; fcasfer@upo.es; jordigarcia@ub.edu

'Departament de Genètica and Institut de Biomedicina (IBUB), Universitat de Barcelona, Barcelona, Spain

${ }^{2}$ Centro Andaluz de Biología del Desarrollo (CABD), CSIC-Universidad Pablo de Olavide. Campus UPO, Ctra. de Utrera km1, E-41013 Sevilla, Spain Full list of author information is available at the end of the article
}

diffusible signaling factors that determine the further development of the adjacent cellular compartments. ZLI cells characteristically secrete $\operatorname{Shh}[1,2]$, whereas the IsO typically releases Fgf8 and Wnt1 [3].

Bona fide IsO and ZLI organizers are present in all vertebrates, including basal living agnathans $[4,5]$; however, the absence of the key morphogens at analogous topological positions [6-9] suggests that comparable signaling centers are not present in invertebrates with a central nervous system (CNS), including amphioxus, a basal chordate considered to be the best living proxy to the vertebrate-invertebrate ancestor [5]. Like vertebrates, amphioxus has a dorsal hollow neural tube that forms from a neural plate. However, the amphioxus brain is relatively simple, consisting only of a putative non-subdivided diencephalon, a Hox-patterned hindbrain and perhaps a small midbrain [10].

Despite the lack of internal brain organizers in invertebrates, previous observations suggested that the interface between the abutting expression domains of the 


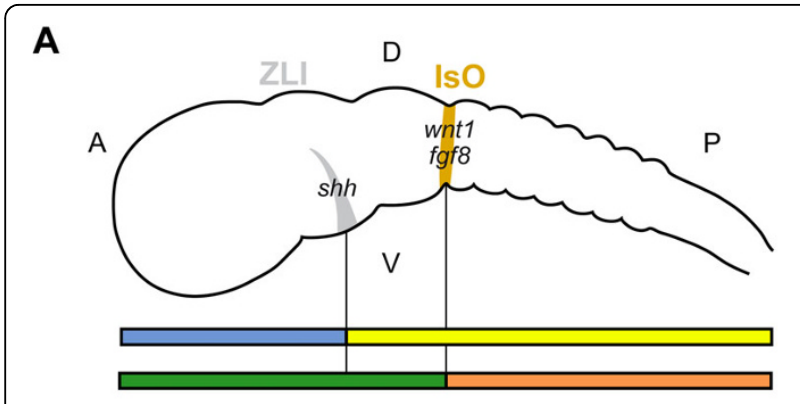

B

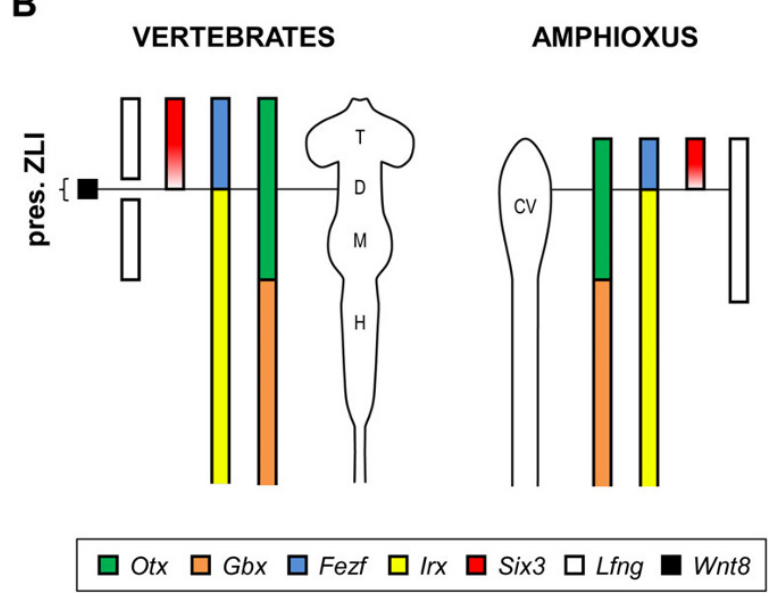

Figure 1 Schematic view of a generalized vertebrate CNS. (A) Topological location of the $\mathrm{ZLI}$ and $\mathrm{IsO}$ organizers and the key genes determining their position in a schematic view of a generalized developing vertebrate central nervous system. The most anterior of the internal brain secondary organizers, the ZLI, develops within the diencephalon, at the interface of the expression of Fezf (blue) and Irx (yellow). The IsO organizer will be located at the midbrain hindbrain boundary (MHB), delimited by the expression of members of the Otx (green) and Gbx (orange) gene families. (B) Scheme of comparative gene expression domains in schematic representations of (left) prototypical vertebrate and (right) amphioxus larva brains (based on [58]). Whereas some patterning genes involved in ZLI location have a conserved relative expression pattern, others like Lfing/Fng (white) or Wnt8 (black) do not. CV = cerebral vesicle; $D=$ diencephalon; $H=$ hindbrain; $M=$ midbrain; $\mathrm{T}=$ telencephalon. Note that adult/larval brain structures from vertebrates and amphioxus are not fully comparable.

Otx/otd and Gbx/unpg genes, which determines the positioning of the MHB (Figure 1), is ancestral to all bilaterians [11,12]. By contrast, much less is known about the evolutionary origin of the other major anterior-posterior brain subdivision and the ZLI, and how the two organizers physically related to each other originally. Recently, the zinc-finger Fezf gene family was reported to have a primary role in establishing the ZLI in vertebrates [13-15]. Fezf is expressed exclusively in the most anterior part of the brain, and its caudal expression abuts that of Irx genes. The interface of the Fezf and Irx expression domains delineates the border between the prethalamus and thalamus at the vertebrate diencephalon, and the position at which the ZLI will develop (Figure 1A). However, no studies have yet investigated the origin of the ZLI in organisms other than vertebrates. To gain insights into these questions, we characterized the Fezf gene in the basal chordate amphioxus and in the protostome Drosophila melanogaster. We analyzed their developmental expression patterns and compared them with those of the Irx, Otx and $G b x$ genes. Strikingly, we found that the relative expression of Fezf, Irx, Otx and Gbx genes in the CNS is fully conserved between these species, suggesting a widespread involvement of these genes in early molecular patterning of the bilaterian CNS.

\section{Results and Discussion}

\section{Fezf is highly conserved across phyla and is ancestral to} bilaterians

Fezf is a transcription factor of the $\mathrm{C} 2 \mathrm{H} 2$ zinc finger family, containing six zinc fingers. Using in silico analysis, we identified putative Fezf orthologs in all studied metazoans (see Methods), including non-bilaterians. The orthology of the different putative Fezf genes is robustly supported by phylogenetic analysis (Figure 2A). The coding sequences of the zinc finger domain are highly conserved between different groups, showing typically $>75 \%$ identity at the amino acid level. In addition to the zinc finger domain, non-bilaterians Fezf proteins have a co-repressor SNAG domain, typical of other related zinc finger gene families, such as Snail and Gfi [16]. This domain was probably present at the origin of Fezf gene family, but has been lost in all studied bilaterians, with the exception of amphioxus, for which we could identify a putative SNAG domain in silico, although reverse transcription PCR experiments showed that this domain is not included in the Fezf transcripts during development of either Branchiostoma floridae or Branchiostoma lanceolatum. Multiple convergent secondary losses of the SNAG domain have also been reported in the Snail/Scratch superfamily [16,17] and it has been proposed that these losses are associated with the acquisition of different conserved domains that carry out a co-repressor function, such as the CtBP-binding site or the NT box [16]. Consistent with this hypothesis, we identified a highly conserved sequence motif near the N-terminus of all studied bilaterian Fezf proteins, which we have termed 'Fezf box' (Figure 2B) and which seems to be exclusive to the Fezf gene family. The clear inverse relation between the presence of the Fezf box (in bilaterians) and the SNAG domain (in non-bilaterians and related genes) suggests that this previously unidentified conserved motif might also function as a co-repressor domain. 


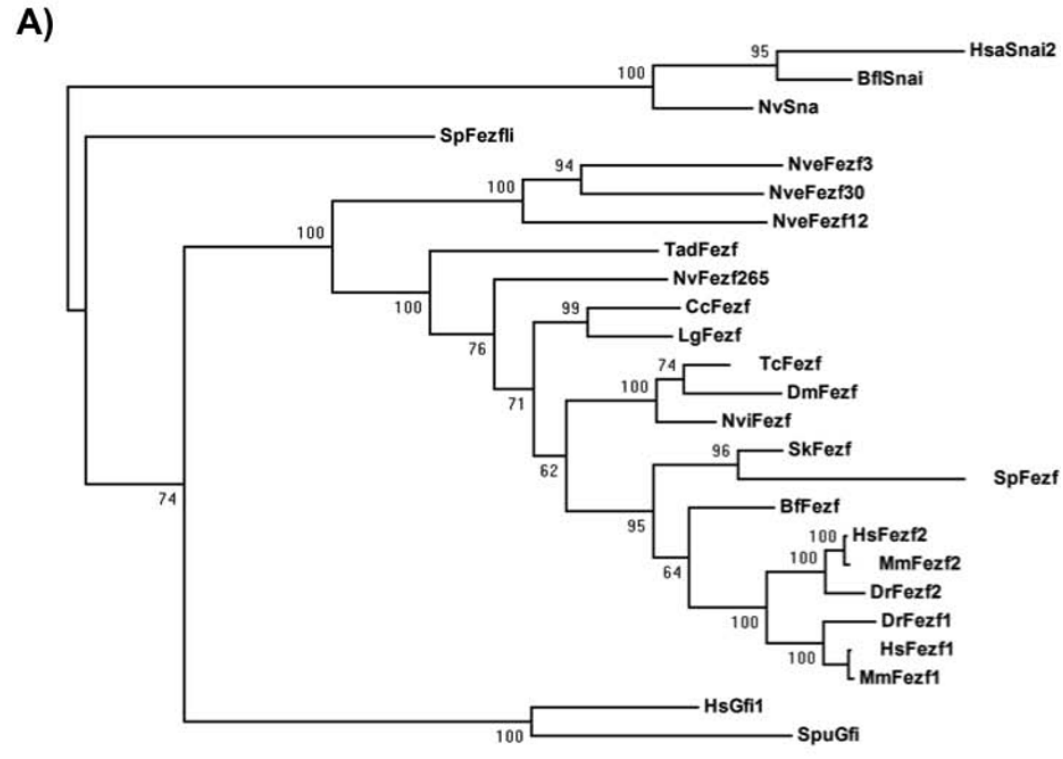

B)

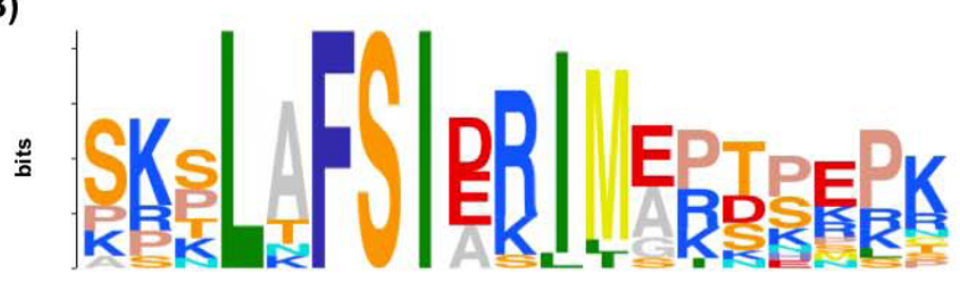

C)

\begin{tabular}{|c|c|c|}
\hline HSAFEZF1 & KPKVFTCEV * CGKV 0 & $\mathrm{EN}$ \\
\hline DreFezf1 & KPKVFTCEV * CGKV 0 & FNAHYI \\
\hline HSAFEZF2 & KPKNFTCEV * CGKV 0 & \\
\hline DreFezf2 & KPKNFTCEV * CGKV 0 & \\
\hline Cinfezf & RKNNFSCGI 0 CGKT * & FKA \\
\hline BflFezf & KQKTFPCEV * CGKI * & \\
\hline SpuFezf & KPKTFVCSE * CGKI & \\
\hline iFezf & SQKTFTCQE * CGKV & \\
\hline neFezf & KPKTFSCLE * CGKV & FNE \\
\hline veFezf1 & TPKTETCEV * CGKV & \\
\hline HSAFEZF1 & RSSTLNTHTRIHAGYKPF & VCEEC \\
\hline EFezf1 & RSSTLNTHTRIHAGYKPE & \\
\hline HSAFEZF2 & RSSTLNTHIRIHAGYKPF & VCEF \\
\hline EFezf2 & RSSTLNTHIRIHAGYKP & CEE \\
\hline Cinfezf & RSSTLNTHMRIHTGLRPY & $\mathrm{CCDI}$ \\
\hline Bf1Fezf & RSST LNTHMR I HAGYKPE & \\
\hline SpuFezf & RSSTLNTHMRIHADFKPE & ICEF \\
\hline LgiFezf & RSSTLNTHMRIHNGYKPE & TCEY \\
\hline DmeFezf & RSSTLNTHSRIHAGYKPF & VCEY \\
\hline NveFezf1 & RSSTLNTHIRIHAGYKE & QCDI \\
\hline 1 & ICPTCGKG & IVRKI \\
\hline DreFezf1 & TCPTCGKGFCRNFDLKKH & IRKL. \\
\hline HsaFezf2 & TCATCGKGFCRNFDLKKH & VRKLH \\
\hline DreFezf2 & TCGTCGKGFCRNFDLKKH & \\
\hline Cinfezf & TCTVCGKGFCRNFDLKKH & AARK \\
\hline BflFezf & TCDICGKGFCRNFDLKKH & IRKLH \\
\hline SpuFezf & TCGICGKGFCRNFDLKKH & TRRKL: \\
\hline LgiFezf & TCVICGKGFCRNFDLKKH & IRKLH \\
\hline DmeFezf & TCRVCAKGFCRNFDLKKH & \\
\hline NveFezf1 & TCLECGKGFCRNFDLKKH & IVRKI \\
\hline
\end{tabular}

Figure 2 Phylogenetic relationships, Fezf box, and exon-intron structure of Fezf genes across metazoans. (A) Phylogenetic tree of the putative Fezf orthologs identified in different metazoan genomes generated by Bayesian inference. The orthology of the genes is supported by a posterior probability of 1. (B) Consensus sequence of the conserved Fezf box located near the C-terminal of all bilaterian Fezf orthologs. (C) Alignment of the zinc finger domains of some representative species showing intron positions and intron phases (colored numbers). Only vertebrates and Ciona intestinalis show lineage-specific introns in these domains. 
In addition to the canonical Fezf genes, we also found 'Fezf-like' genes in cnidarians and sea urchin (NveFezf3, NveFezf12 and NveFezf30 and SpuFezli [18]); these genes branch at basal positions of the phylogenetic tree (Figure 2A) and contain a SNAG domain but not a Fezf box.

Finally, we also studied the exon-intron structure within the zinc finger domain, where the sequence can be confidently aligned. Surprisingly, whereas in nearly all species no introns are found within the zinc finger domain, all vertebrate genes have three introns (one conserved with the tunicate Ciona) that seem to be lineage-specific gains (Figure 2C). This is unexpected, considering the high conservation of intron positions from cnidarians to vertebrates in the deuterostome line [19-23] and the generally low rate of intron gains along these lineages.

\section{Expression of Fezf, Irx and Gbx in amphioxus}

To gain insights into the evolutionary origin of the vertebrate ZLI, we analyzed the developmental expression of the single Fezf gene of the basal chordate amphioxus (B. lanceolatum). As in vertebrates, Fezf expression starts at the beginning of neurulation, and its expression is highly restricted to the most anterior part of the neural plate (the six to seven anterior-most rows of cells) (Figure 3A, B). This restricted anterior neural domain continues to the larval stages (Figure $3 \mathrm{C}, \mathrm{D}$ ), at which point the expression is found only in the cerebral vesicle, the most anterior part of the amphioxus larval neural tube. We next compared Fezf expression to Irx and $G b x$ genes at neurula stage, which in vertebrates mark the posterior boundaries of the presumptive ZLI and $\mathrm{MHB}$, respectively. Strikingly, we found that the relative expression of Fezf, Irx and Gbx genes in the neural plate is fully conserved between amphioxus and vertebrates (Figure 4A, B), indicating that these genetic interfaces, which contribute to delineate these major brain subdivisions, were present before the origin of vertebrates. In both Xenopus and amphioxus, the expression of Fezf abuts that of Irx, and there is a conserved gap between the expression domains of Fezf and Gbx that shows Irx expression (Figure 4A, B), consistent with an ancestrally conserved anterior-posterior topology of the MHB positioning relative to the ZLI. Moreover, the abutting expression of the Fezf and Irx genes constitutes a conserved genetic subdivision within the amphioxus presumptive diencephalon, raising the intriguing possibility of potential equivalents of proto-prethalamic and a proto-thalamic regions in the primitive chordate brain, consistent with other observations [24,25]. Further investigation will be required to assess to what extent these structures are homologous and functionally equivalent to their vertebrate counterparts or whether they correspond to distinct amphioxus novelties.

\section{Expression of Fezf in flies}

To further investigate the evolutionary origin of these early genetic brain subdivisions, we examined the expression patterns of Fezf and Irx homologous in the Drosophila developing CNS. dFezf/Earmuff/CG31670 has been recently shown to maintain the restricted developmental potential of intermediate neural progenitors in Drosophila [26], and its embryonic expression pattern has been documented previously [27]. As in chordates, dFezf expression is restricted to the most anterior part of the fly CNS throughout early CNS development (Figure 3E-J). Fezf expression starts in blastoderm embryos as a dorsal and lateral stripe in the anterior (neurogenic procephalic) region of the blastoderm (Figure 3E,F). Characteristically, the lateral ends of this stripe widen, making the pattern resemble earmuffs (Figure 3G). In early germband extension-stage embryos, the stripe is split at the dorsal midline (Figure 3G), generating bilaterally symmetrical domains (Figure 3G-J). During later embryogenesis, $d F e z f$-expressing cells delaminate and cluster to form part of the brain hemispheres (Figure 3H-J). Importantly, the expression domain of mirror (mirr), the earliest fly Irx expressed gene, also abuts that of $d F e z f$ (Figure 4C). Our results, along with the fact that in Drosophila the orthologs of $O t x$ and $G b x$ also show complementary expression domains (Figure 4C, [12]), and the presence of the conserved gap between the expression domains of fly Fezf and $G b x$ orthologs, suggest that this simple initial genoarchitectural plan, which broadly subdivides the vertebrate nervous system, was present in the last common ancestor of extant bilateral animals.

Significantly, Fezf and Irx in vertebrates regulate each other in a mutually exclusive manner. In knockout or knockdown mutants for these genes in different vertebrate species, there is a shift in the expression limit of the counterpart gene, either anteriorly (in the case of Irx $[13,14]$ ) or posteriorly (in the case of Fezf [15]). To assess whether this situation was at least partially conserved in flies, we also analyzed the expression of $d F e z f$ in iro ${ }^{D F M 3}$ mutant embryos, which lack the $\operatorname{Irx}$ genes [28]. In stark contrast to vertebrates, we did not find any noticeable caudal shift in the posterior limit of $d F e z f$ expression (data not shown).

\section{Early complex brains}

The strongly restricted expression of Fezf to the anterior forebrain in vertebrates is an indication of its crucial role in the patterning of the vertebrate brain. The presence of deeply conserved Fezf orthologs in all studied 

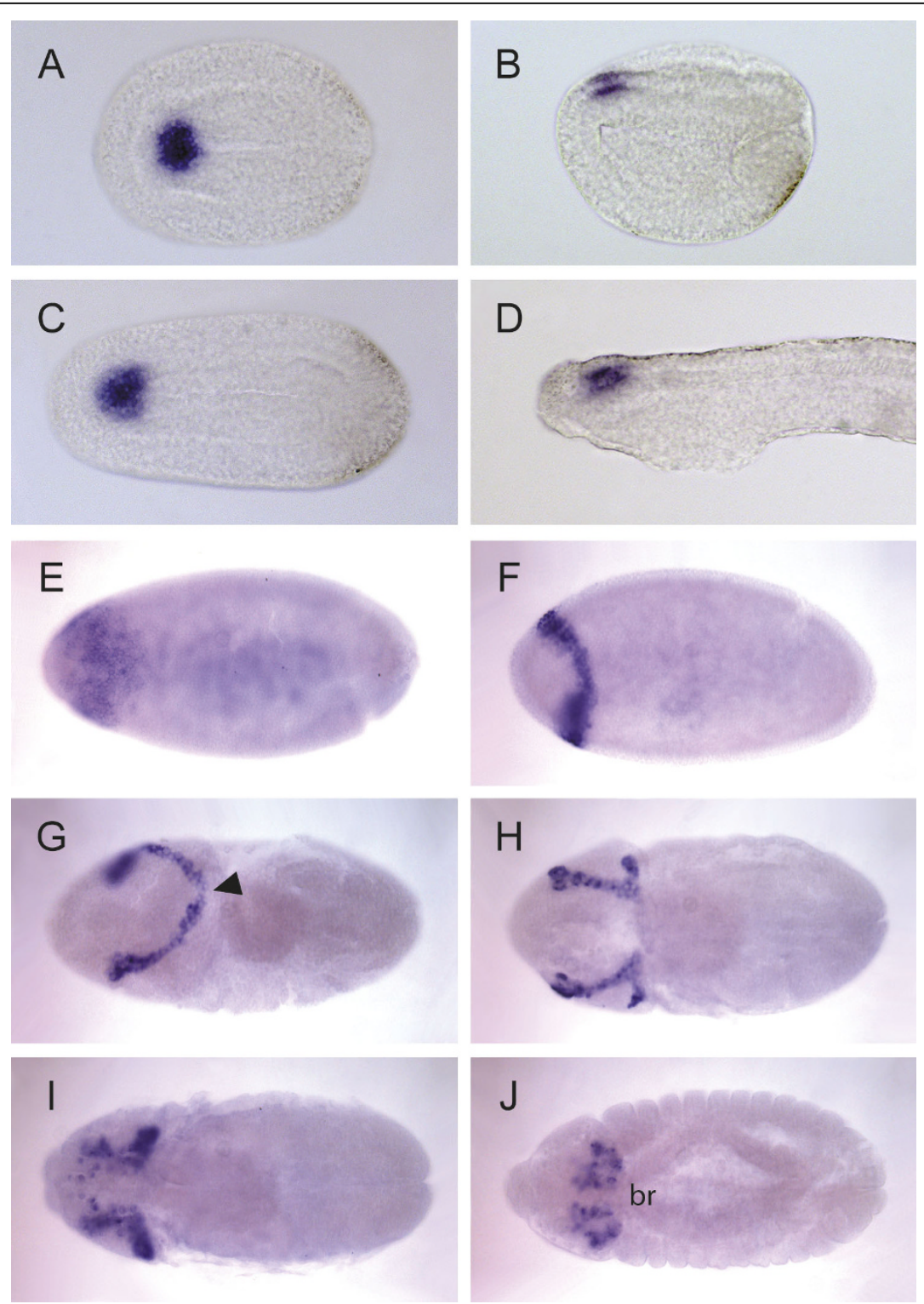

Figure 3 Developmental expression of Fezf in amphioxus and Drosophila. (A-D) Expression of Branchiostoma lanceolatum Fezf (purple) during embryogenesis. (A) Dorsal view of an early neurula showing the highly restricted Fezf expression (purple) in the anterior neural plate. Expression is observed only in the anterior-most six to seven rows of cells in the neural plate. (B) Side view of the same stage shown in (A). (C) Dorsal view of a mid-neurula stage, showing similarly strongly restricted anterior neural expression. (D) Lateral view of a premouth larva. Anterior is to the left and dorsal is up. (E-J): Expression of Drosophila Fezf (purple) during embryogenesis, as previously reported [27], in embryos at stages 4 (syncitial blastoderm), 5 (cellular blastoderm), 8, 10, 11-12 and 14, respectively, as described previously [59]. Anterior is to the left. (E, F, H-J) dorsal views; (G) dorsolateral view. Arrowhead in (G) indicates the dorsal split of the initially continuous stripe. br brain hemispheres.

metazoans, from placozoans to vertebrates, thus raises the question of whether Fezf might play a similar conserved role throughout animal phylogeny, or whether it has been recruited for different developmental functions in the different phyla. We show that in two distantly related invertebrate groups with a centralized CNS Fezf orthologs are also expressed in a strongly restricted manner in the developing anterior CNS, suggesting that the ancestral function of Fezf in bilaterians might well be related to the patterning of the CNS. Furthermore, the conserved relative expression with other key patterning genes (Irx, Gbx and Otx) at early neurulation stages suggest that all these genes may help to define broad conserved regions within the neural ectoderm as a whole in different bilaterian organisms [11,12,29,30].

Based on several similarities in patterning gene expression and function, Reichert and collaborators suggested that the last common ancestor of extant bilaterians, 


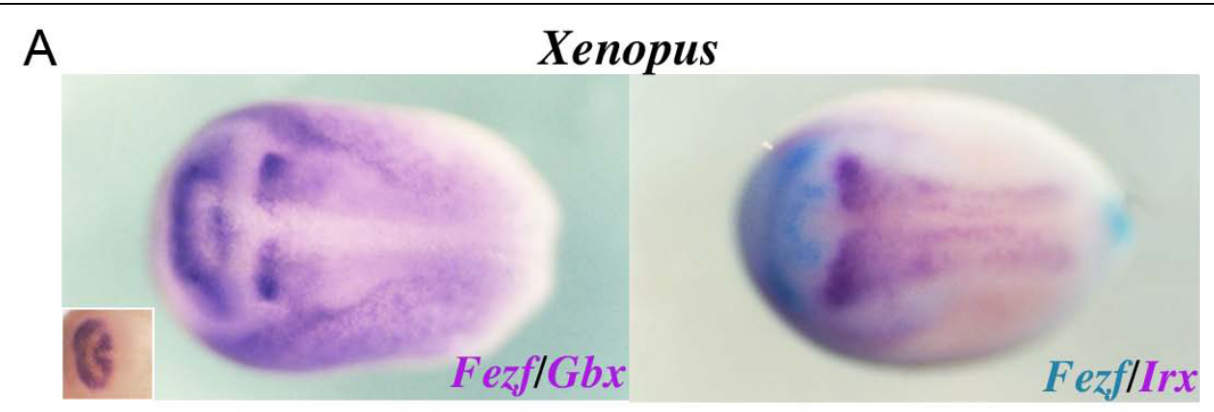

B

Amphioxus

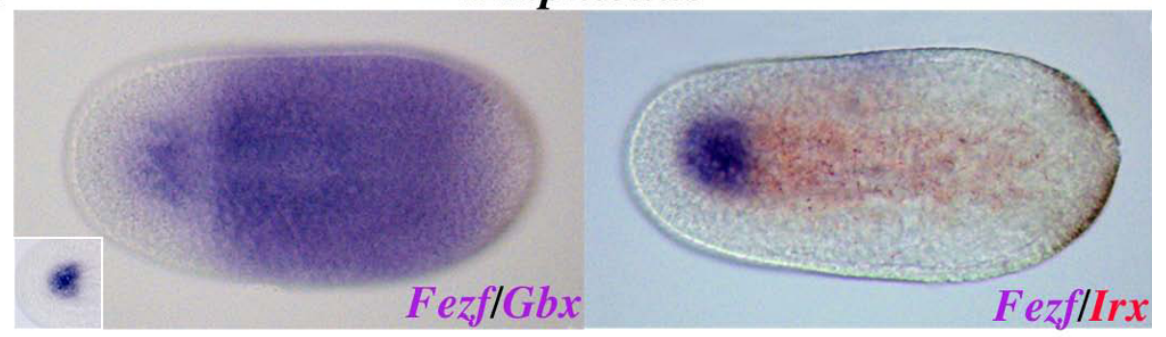

C

Drosophila
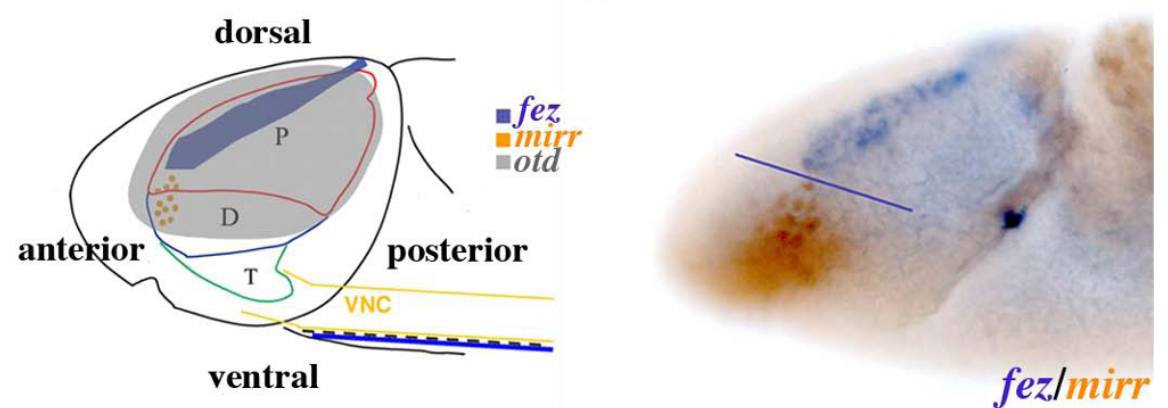

Figure 4 Conserved basic CNS genoarchitecture in bilaterians. (A) Expression of Fezf and Gbx2 in Xenopus neurula showing a gap between the expression of both genes (left) at the neural plate and expression of Fezf (blue) and Irxl (purple) showing (right) abutting expression

domains. (B) Expression of Fezf and Gbx in (left) the amphioxus neurula and expression of Fezf (purple) and IrxB (red) showing (right) abutting expression domains. (C) Schematic representation of Drosophila CNS (protocerebrum (P), deutocerebrum (D) and tritocerebrum (T) correspond in vertebrates with the forebrain, midbrain and hindbrain, respectively) showing the expression of (left) the dFezf (earmuff/CG31670), Irx (mirr) and Otx (otd) (Gbx (unpg) is expressed latter abutting Otx [12], and lateral view of the expression patterns of Fezf (purple) and mirr (orange). Anterior is to the left, dorsal views unless otherwise specified. Insets in (A) Xenopus and (B) amphioxus correspond to single in situ hybridization of Fezf.

Urbilateria, had a tripartite brain, and that Drosophila and vertebrate brains had a comparable anterior-posterior patterning [12,31,32]. These authors proposed a model with three domains consisting (from anterior to posterior) of: (1) forebrain/midbrain, (2) an intervening $\mathrm{MHB}$ region and (3) a hindbrain. These three structures are characterized by the specific expression of the Otx, Pax 2/5/8 and Hox genes, respectively [12,31]. Our results complement and expand this model, adding an extra conserved genetic subdivision to the forebrain/protocerebrum of the studied species.

\section{Origin of the ZLI secondary organizer}

In addition to Fezf-Irx, other sets of genes with mutually exclusive expression patterns have been proposed to be involved in the development of the ZLI in vertebrates. Based on misexpression analysis, it was first suggested that the mutual repression between Irx genes and Six3 (expressed at early stages in the whole anterior forebrain anlage, limited caudally by the anterior boundary of Irx genes) (Figure 1B[33]) contributed to the establishment of the ZLI and other diencephalic subdivisions [34]. In amphioxus early neurula, BfSix $3 / 6$ is expressed in the anterior-most part of the neural plate, with a posterior boundary seemingly consistent with that of Fezf-Irx [24]. However, the role of Six3 in establishing the ZLI has recently been challenged, because, in contrast to Fezf, Six3 expression is very dynamic and regresses rostrally, both in vertebrates and amphioxus [24,33,34], leaving a region free of Irx and Six3. Six 3 function might thus be 
related to cell proliferation rather than to neural patterning at these stages [24].

Another important pair of genes with mutually exclusive expression patterns thought to be involved in ZLI formation in vertebrates are Lfng and Wnt8b [1]. Lfng is expressed widely in the chick prosencephalon, with the exception of a wedge-shaped area (presumptive ZLI), where $W n t 8 b$ is expressed (Figure 1B, [1]). This Wnt8bpositive/Lfng-negative region is where the ZLI will develop. In amphioxus, however, the single BfFng gene is expressed throughout the anterior-most part of the neural plate in early neurula, apparently with no discontinuity [35], whereas $B f W n t 8$ is not expressed in the CNS at early developmental stages [36] (Figure 1B).

Taken together, these results suggest that, although the genetic boundary determining the location of the ZLI in vertebrates was present in proximal chordate ancestors, some of the components of the putative ZLI gene network [1] were not yet assembled. Accordingly, molecules secreted as secondary organizers in vertebrates have not been found in the amphioxus developing brain $[7,9]$. Thus, it is likely that in vertebrate ancestors, the ZLI secondary organizers evolved through the recruitment of the expression of key morphogens to the cells located in the interface of these conserved major genetic domains, defined by the abutting expressions of Fezf-Irx. Presumably, this would have led to the development of new subdivisions and brain structures, possibly allowing the increase in proliferation and complexity of present-day vertebrate brains. However, it is also possible that internal brain organizers evolved before the vertebrates originated, and were then lost in the studied invertebrate chordates by reductive evolution. Equivalent signaling centers have not been reported in any invertebrate to date; however, it is still possible that a more thorough study of other invertebrates, such as hemichordates, which show a wide conservation of relative gene expression patterns with vertebrates [37], or basal slow-evolving protostomes, for which there are no molecular data yet available, will help to clarify the origins of the vertebrate brain complexity.

\section{Methods}

\section{In silico Identification and comparison of Fezf genes across metazoans}

Using the previously described vertebrate Fezf genes as queries, we performed tBLASTN and/or BLASTP searches against the genomes of Branchiostoma floridae JGI v1.0, Trichoplax adhaerens Grell-BS-1999 v1.0, Nematostella vectensis JGI v1.0, Ciona intestinalis JGI v2.0, Daphnia pulex JGI v1.0, Lottia gigantea JGI v1.0 and Capitella teleta JGI v1.0, using the JGI website (http://genome.jgi-psf.org/euk_home.html) and of Strongylocentrotus purpuratus Build 2.1, Tribolium castaneum
Build 2.1, Nasonia vitripennis Build 1.1, Drosophila melanogaster Build Fb5.3, Homo sapiens Build GRCh37, Mus musculus Build 37.1, Danio rerio Build Zv8, using the NCBI website (http://www.ncbi.nlm.nih.gov/blast/ Blast.cgi). For Saccoglossus kowalevskii we performed a tBLASTN search against the traces at NCBI and then manually assembled the genomic locus.

We then downloaded each corresponding genomic region and build different gene models using GenomeScan [38] and GeneWise2 [39] software as necessary. We compared these predictions with expressed sequence tags and existent gene models when available. Annotation and comparison of intron positions and phases across zinc finger domains was performed as previously described $[40,41]$.

The amino acid sequences for the zinc finger domains were aligned using ClustalW [42] and the resulting alignment was manually curated. Phylogenetic trees were then generated by the Bayesian method, using the software MrBayes 3.1.2 [43,44], with the model Dayhoff +Gamma, recommended by ProtTest 1.4 [45-47], under the Akaike information and the Bayesian information criterions. Two independent runs were performed, each with four chains. For convention, convergence was reached when the value for the standard deviation of split frequencies stayed below 0.01. Burn-in was determined by plotting parameters across all runs for a given analysis: all trees before stationarity and convergence were discarded, and consensus trees were calculated for the remaining trees (from at least 1,000,000 generations).

Fezf box consensus was decided by the program Sequence Logo online (http://genome.tugraz.at/Logo/) using a multiple alignment for all studied species containing a Fezf box.

\section{Cloning of European amphioxus Fezf, Irx and Gbx genes}

Primer pairs were designed to span the whole length coding sequences of the B. floridae Fezf [18] and Gbx [11] genes, if possible. A liquid cDNA library from different developmental stages of the European amphioxus (Branchiostoma lanceolatum) was screened by PCR using the B. floridae Fezf primers. B. lanceolatum Fezf and $G b x$ were cloned, sequenced and submitted to NCBI (accession numbers HM245959, HM245960; primer sequences: Fezf_L: ATGGCAATGTTCGGA ACCCTTG, Fezf_R: TTACTCTGCGGCTGGAAGTG, Gbx_L: TGAAAATGCAGCGGCACAGC, Gbx_R: ATGCTGACTCCTCATGGCGAA). For BlIrxB, we used the previously reported full-length sequence [48]. Neural plate expression patterns for Irx and Gbx in B. lanceolatum were consistent with those reported in B. floridae $[11,29]$. To assess whether the putative SNAG domain was included in the transcripts we used the following 
primers: GCGACGGTTCCATAATTCGT (reverse, within the CDS) with M13F standard primer (for cDNA library amplifications) or ATGCCAAAGTCATTTC TGGTG (in the predicted SNAG domain) and. All bands were cloned and sequenced. A liquid cDNA library of B. floridae provided by G. Langeland and our own cDNA library of $B$. lanceolatum were used as templates.

\section{In situ hybridization in the different species, antibody staining and Drosophila strains}

Antisense RNA probes were prepared from cDNAs using digoxigenin or fluorescein (Boehringer Mannheim $\mathrm{GmBH}$, Mannheim, Germany) as labels. The Drosophila Fezf cDNA (GH 14092) corresponding to the CG31670 was obtained from the Drosophila Genome Resources.

Xenopus specimens were prepared, hybridized and stained as previously described $[49,50]$. For in situ hybridization of European amphioxus, we used a modified version of the protocol previously described [51] (see Additional file 1). Importantly, the hybridization temperature was $65^{\circ} \mathrm{C}$, and antibodies were incubated for 3 to 4 hours, followed by overnight washes in MABT buffer ( $100 \mathrm{mM}$ maleic acid, $150 \mathrm{mM} \mathrm{NaCl}, 0.1 \%$ Tween$20, \mathrm{pH} 8)$ to reduce background. Detection was done with alkaline phosphatase-conjugated anti-digoxigenin (DIG) or anti-fluorescein antibodies. Alkaline phosphatase reaction products were visualized with nitroblue tetrazolium chloride (NBT)-5-bromo-4-chloro-3'-indolyphosphate $p$-toluidine salt (BCIP) (purple color), 2-(4iodophenyl)-5-(4-nitro-phenyl)-3-phenyltetrazolium chloride (INT)-BCIP (red) or BCIP only (cyan). Drosophila embryos were collected on yeasted apple juiceagar plates [52]. Pretreatment of embryos and hybridization in situ were performed as previously described [53], with some modifications: proteinase $\mathrm{K}$ treatment was avoided and incubations with anti-DIG (1:1000) were performed for 1 hour at room temperature. For double in situ hybridization and inmunostaining, the rabbit anti- $\beta$-galactosidase (Cappel) antibody was incubated with the anti-DIG. First, $\beta$-galactosidase detection was carried out as described previously [54], then the in situ hybridization signal was developed as described above. The Drosophila strain used were mirr $^{880-l a c Z}$ and the Irx deficiency iro $^{D F M 3}[55,56]$. The deficiency iro ${ }^{D F M 3}$ was balanced over the 'blue' balancer TM6B, P $\{35 \mathrm{UZ}\} \mathrm{DB} 1$, $\mathrm{Tb}^{1}$ (Flybase: http://flybase.org/). Embryos were simultaneously hybridized with probes against $d F e z f$ and anti $\beta$ galactosidase transcripts, and homozygous iro ${ }^{D F M 3}$ embryos were those not transcribing $\beta$-galactosidase. Embryos were dehydrated and mounted as previously described [57].

\section{Additional material}

Additional file 1: Branchiostoma lanceolatum ISH protocol. Detailed protocol used for whole-mount in situ hybridization in the European amphioxus B. lanceolatum embryos

\section{Acknowledgements}

We thank Senda Jimenez-Delgado for help on the experimental work, Jose Luis Ferrán for helpful comments and discussions, and Isabel Almudí for help on image processing. MI, IM and JGF were funded by grants BFU200500252 and BMC2008-03776 from the Spanish Ministerio de Educación y Ciencia (MEC), MI and IM hold FPI and FPU fellowships, respectively and JGF the ICREA Academia Prize; CP and FC were funded by grants BFU200600349/BMC (MEC) and CVI 2658 (Junta de Andalucía) and JLGS by grants BFU2007-60042/BMC, Petri PET2007_0158, CSD2007-00008 (MEC) and CVI 3488 (Junta de Andalucía).

\section{Author details}

${ }^{1}$ Departament de Genètica and Institut de Biomedicina (IBUB), Universitat de Barcelona, Barcelona, Spain. ${ }^{2}$ Centro Andaluz de Biología del Desarrollo (CABD), CSIC-Universidad Pablo de Olavide. Campus UPO, Ctra. de Utrera km1, E-41013 Sevilla, Spain.

\section{Authors' contributions}

$\mathrm{Ml}$ conceived the study, carried out the expression experiments in amphioxus and participated in the sequence analyses. CP generated the Drosophila data. IM participated in the sequence analyses and performed the Xenopus and amphioxus ISH. JLGS conceived and participated in the design and coordination of the experiments and generated Xenopus data. FC coordinated the Drosophila experiments. JGF participated in the design and coordination of the project. MI, JLGS, FC and JGF wrote the draft manuscript, and all authors read, discussed and approved the manuscript.

\section{Competing interests}

The authors declare that they have no competing interests.

Received: 11 March 2010 Accepted: 1 September 2010

Published: 1 September 2010

\section{References}

1. Kiecker C, Lumsden A: Compartments and their boundaries in vertebrate brain development. Nat Rev Neurosci 2005, 6:553-564.

2. Scholpp S, Wolf O, Brand M, Lumsden A: Hedgehog signalling from the zona limitans intrathalamica orchestrates patterning of the zebrafish diencephalon. Development 2006, 133:855-864.

3. Martinez S: The isthmic organizer and brain regionalization. Int J Dev Biol 2001, 45:367-371.

4. Osorio J, Mazan S, Retaux S: Organisation of the lamprey (Lampetra fluviatilis) embryonic brain: Insights from LIM-homeodomain, Pax and hedgehog genes. Dev Biol 2005, 288:100-112.

5. Murakami Y, Uchida K, Rijli FM, Kuratani S: Evolution of the brain developmental plan: Insights from agnathans. Dev Biol 2005, 280:249-259.

6. Lowe CJ, Terasaki M, Wu M, Freeman RM, Runft L, Kwan K, Haigo S, Aronowicz J, Lander E, Gruber C, Smith M, Kirschner M, Gerhart J: Dorsoventral patterning in hemichordates: insights into early chordate evolution. PLOS Biol 2006, 4:e291.

7. Shimeld SM: The evolution of the hedgehog gene family in chordates: insights from amphioxus hedgehog. Dev Genes and Evol 1999, 209:40-47.

8. Meulemans D, Bronner-Fraser M: Insights from amphioxus into the evolution of vertebrate cartilage. PLOS One 2007, 2:e787.

9. Holland LZ, Short S: Gene duplication, co-option and recruitment during the origin of the vertebrate brain from the invertebrate chordate brain. Brain Behav Evol 2008, 72:91-105.

10. Holland LZ, Satoh N, Azumi K, Benito-Gutiérrez Ė, Bronner-Fraser M, Brunet F, Butts T, Candiani S, Dishaw LD, Ferrier DEK, Garcia-Fernàndez J, 
Gibson-Brown JJ, Gissi C, Godzik A, Hallbrook F, Hirose D, Hosomichi K, Ikuta T, Inoko H, Kasahara M, Kasamatsu J, Kawashima T, Kimura A, Kobayashi M, Kozmik Z, Kubokawa K, Laudet V, Litman GW, Mchardy AC, Meulemans $D$, et al: The amphioxus genome illuminates vertebrate origins and cephalochordate biology. Genome Res 2008, 18:1100-1111.

11. Castro LFC, Rasmussen SLK, Holland PWH, Holland ND, Holland LZ: A Gbx homeobox gene in amphioxus: Insights into ancestry of the ANTP class and evolution of the midbrain/hindbrain boundary. Dev Biol 2006, 295:40-51.

12. Hirth F, Kammermeier L, Frei E, Walldorf U, Noll M, Reichert H: An urbilaterian origin of the tripartite brain: developmental genetic insights from Drosophila. Development 2003, 130:2365-2373.

13. Hirata T, Nakazawa M, Muraoka O, Nakayama R, Suda Y, Hibi M: Zinc-finger genes Fez and Fez-like function in the establishment of diencephalon subdivisions. Development 2006, 133:3993-4004.

14. Jeong J-Y, Einhorn Z, Mathur P, Chen L, Lee S, Kawakami K, Guo S: Patterning the zebrafish diencephalon by the conserved zinc-finger protein Fezl. Development 2007, 134:127-136.

15. Rodríguez-Seguel E, Alarcón P, Gómez-Skarmeta JL: The Xenopus Irx genes are essential for neural patterning and define the border between prethalamus and thalamus through mutual antagonism with the anterior repressors Fezf and Arx. Dev Biol 2009, 329:258-268.

16. Barrallo-Gimeno A, Nieto MA: Evolutionary history of the Snail/Scratch superfamily. Trends Genet 2009, 25:248-252.

17. Kerner $P$, Hung J, Béhague J, Le Gouar M, Balavoine G, Vervoort M: Insights into the evolution of the snail superfamily from metazoan wide molecular phylogenies and expression data in annelids. BMC Evol Biol 2009, 9:94.

18. Shimeld SM: $\mathrm{C} 2 \mathrm{H} 2$ zinc finger genes of the Gli, Zic, KLF, SP, Wilms' tumour, Huckebein, Snail, Ovo, Spalt, Odd, Blimp-1, Fez and related gene families from Branchiostoma floridae. Dev Genes Evol 2008, 218:639-649.

19. Sullivan JC, Reitzel AM, Finnerty JR: A high percentage of introns in human genes were present early in animal evolution: evidence from the basal metazoan Nematostella vectensis. Genome Inform 2006, 17:219-229.

20. Coulombe-Huntington J, Majewski J: Characterization of intron loss events in mammals. Genome Res 2007, 17:23-32.

21. Roy SW, Fedorov A, Gilbert W: Large-scale comparison of intron positions in mammalian genes shows intron loss but no gain. Proc Natl Acad Sci USA 2003, 100:7158-7162.

22. Putnam NH, Srivastava M, Hellsten U, Dirks B, Chapman J, Salamov A, Terry A, Shapiro H, Lindquist E, Kapitonov W, Jurka J, Genikhovich G, Grigoriev IV, Lucas SM, Steele RE, Finnerty JR, Technau U, Martindale MQ, Rokhsar DS: Sea anemone genome reveals ancestral eumetazoan gene repertoire and genomic organization. Science 2007, 317:86-94.

23. Putnam N, Butts T, Ferrier DEK, Furlong RF, Hellsten U, Kawashima T, Robinson-Rechavi M, Shoguchi E, Terry A, Yu JK, Benito-Gutiérrez E, Dubchak I, Garcia-Fernàndez J, Grigoriev IV, Horton AV, de Jong PJ, Jurka J, Kapitonov V, Kohara Y, Kuroki Y, Lindquist E, Lucas S, Osoegawa K, Pennacchio LA, Asaf Salamov A, Satou Y, Sauka-Spengler T, Schmutz T, Shin-I T, Toyoda A, et al: The amphioxus genome and the evolution of the chordate karyotype. Nature 2008, 453:1064-1071.

24. Kozmik Z, Holland ND, Kreslova J, Oliveri D, Schubert M, Jonasova K, Holland LZ, Pestarino M, Benes V, Candiani S: Pax-Six-Eya-Dach network during amphioxus development: conservation in vitro but context specificity in vivo. Dev Biol 2007, 306:149-159.

25. Schubert M, Holland LZ, Stokes MD, Holland ND: Three amphioxus Wnt genes (AmphiWnt3, AmphiWnt5, and AmphiWnt6) associated with the tail bud: the evolution of somitogenesis in chordates. Dev Biol 2001, 240:262-273.

26. Weng $\mathrm{M}$, Golden $\mathrm{KL}$, Lee $\mathrm{CY}$ : dFezf/Earmuff maintains the restricted developmental potential of intermediate neural progenitors in Drosophila. Dev Cell 2010, 18:126-135.

27. Pfeiffer BD, Jenett A, Hammonds AS, Ngo TT, Misra S, Murphy C, Scully A, Carlson JW, Wan KH, Laverty TR, Mungall C, Svirskas R, Kadonaga JT, Doe CQ, Eisen MB, Celniker SE, Rubin GM: Tools for neuroanatomy and neurogenetics in Drosophila. Proc Natl Acad Sci USA 2008, 105:9715-9720.

28. Gomez-Skarmeta JL, Diez del Corral R, de la Calle-Mustienes E, FerréMarcó D, Modolell J: Araucan and caupolican, two members of the novel iroquois complex, encode homeoproteins that control proneural and vein-forming genes. Cell 1996, 85:95-105.
29. Kaltenbach SL, Holland LZ, Holland ND, Koop D: Developmental expression of the three iroquois genes of amphioxus (BflrxA, BflrxB, and BflrxC) with special attention to the gastrula organizer and anteroposterior boundaries in the central nervous system. Gene Expr Patterns 2009, 9:329-334.

30. Cavodeassi F, Modolell J, Gomez-Skarmeta JL: The Iroquois family of genes: from body building to neural patterning. Development 2001, 128:2847-2855

31. Reichert $\mathrm{H}$ : A tripartite organization of the urbilaterian brain: developmental genetic evidence from Drosophila. Brain Res Bull 2005, 66:491-494.

32. Lichtneckert $\mathrm{R}$, Reichert $\mathrm{H}$ : Insights into the urbilaterian brain: conserved genetic patterning mechanisms in insect and vertebrate brain development. Heredity 2005, 94:465-477.

33. Seo HC, Drivenes Ellingsen S, Fjose A: Expression of two zebrafish homologues of the murine Six3 gene demarcates the initial eye primordia. Mech Dev 1998, 73:45-57.

34. Kobayashi D, Kobayashi M, Matsumoto K, Ogura T, Nakafuku M, Shimamura K: Early subdivisions in the neural plate define distinct competence for inductive signals. Development 2002, 129:83-93.

35. Mazet F, Shimeld SM: Characterisation of an amphioxus Fringe gene and the evolution of the vertebrate segmentation clock. Dev Genes Evol 2003, V213:505-509.

36. Schubert M, Holland LZ, Panopoulou GD, Lehrach H, Holland ND: Characterization of amphioxus AmphiWnt8: insights into the evolution of patterning of the embryonic dorsoventral axis. Evol Dev 2000, 2:85-92.

37. Lowe CJ, Wu M, Salic A, Evans L, Lander E, Stange-Thomann N, Gruber CE, Gerhart J, Kirschner M: Anteroposterior patterning in hemichordates and the origins of the chordate nervous system. Cell 2003, 113:853-865.

38. Yeh R-F, Lim LP, Burge CB: Computational inference of homologous gene structures in the human genome. Genome Res 2001, 11:803-816.

39. Birney E, Durbin R: Using GeneWise in the Drosophila annotation experiment. Genome Res 2000, 10:547-548.

40. Irimia M, Roy SW: Spliceosomal introns as tools for genomic and evolutionary analysis. Nucleic Acids Res 2008, 36:1703-1712.

41. D'Aniello S, Irimia M, Maeso I, Pascual-Anaya J, Jiménez-Delgado S, Bertrand S, Garcia-Fernàndez J: Gene expansion and retention leads to a diverse tyrosine kinase superfamily in amphioxus. Mol Biol Evol 2008, 25:1841-1854.

42. Higgins DG, Thompson JD, Gibson TJ: Using CLUSTAL for several sequence alignments. Methods Enzymol 1996, 266:383-402.

43. Huelsenbeck JP, Ronquist F: MRBAYES: Bayesian inference of phylogenetic trees. Bioinformatics 2001, 17:754-755.

44. Ronquist F, Huelsenbeck JP: MrBayes 3: Bayesian phylogenetic inference under mixed models. Bioinformatics 2003, 19:1572-1574.

45. Drummond A, Strimmer K: PAL: an object-oriented programming library for molecular evolution and phylogenetics. Bioinformatics 2001, 17:662-663.

46. Abascal F, Zardoya R, Posada D: ProtTest: selection of best-fit models of protein evolution. Bioinformatics 2005, 21:2104-2105.

47. Guindon S, Gascuel O: A simple, fast, and accurate algorithm to estimate large phylogenies by maximum likelihood. Syst Biol 2003, 52:696-704.

48. Irimia M, Maeso I, Garcia-Fernandez J: Convergent evolution of clustering of Iroquois homeobox genes across metazoans. Mol Biol Evol 2008, 25:1521-1525.

49. Harland R: In situ hybridization: an improved whole mount method for Xenopus embryos. Methods Cell Biol 1991, 36:685-695.

50. Tena JJ, Neto A, de la Calle-Mustienes E, Bras-Pereira C, Casares F, GomezSkarmeta JL: Odd-skipped genes encode repressors that control kidney development. Dev Biol 2007, 301:518-531.

51. Yu JK, Holland LZ: Amphioxus whole-mount in situ hybridization. CSH Protoc 2009, 2009, pdb.prot5286.

52. Nusslein-Volhard C: A rapid method for screening eggs from single Drosophila females. Drosophila I\&I Serv 1977, 52:166.

53. Jekely G, Arendt D: Cellular resolution expression profiling using confocal detection of NBT/BCIP precipitate by reflection microscopy. Biotechniques 2007, 42:751-755.

54. Dohrmann C, Azpiazu N, Frasch M: A new Drosophila homeo box gene is expressed in mesodermal precursor cells of distinct muscles during embryogenesis. Genes Dev 1990, 4:2098-2111. 
55. Gómez-Skarmeta JL, Modolell J: Araucan and caupolican provide a link between compartment subdivisions and patterning of sensory organs and veins in the Drosophila wing. Genes Dev 1996, 10:2935-2946.

56. MCNeill H, Yang CH, Brodsky M, Ungos J, Simon MA: Mirror encodes a novel PBX-class homeoprotein that functions in the definition of the dorso-ventral border of the Drosophila eye. Genes Dev 1997, 11:1073-1082

57. Hartenstein V, Posakony JW: The development adult sensilla on the wing and notum of Drosophila melanogaster. Development 1989, 107:389-405.

58. Holland LZ: Chordate roots of the vertebrate nervous system: expanding the molecular toolkit. Nat Rev Neurosci 2009, 10:736-746.

59. Campos-Ortega J, Hartenstein V: The Embryonic Development of Drosophila melanogaster. Heidelberg: Springer-Verlag 1997.

doi:10.1186/2041-9139-1-7

Cite this article as: Irimia et al:: Conserved developmental expression of Fezf in chordates and Drosophila and the origin of the Zona Limitans Intrathalamica (ZLI) brain organizer. EvoDevo 2010 1:7.

\section{Submit your next manuscript to BioMed Central} and take full advantage of:

- Convenient online submission

- Thorough peer review

- No space constraints or color figure charges

- Immediate publication on acceptance

- Inclusion in PubMed, CAS, Scopus and Google Scholar

- Research which is freely available for redistribution

Submit your manuscript at www.biomedcentral.com/submit 